Journal of Chromatography B-

Review articles

\title{
Metabolome analysis for discovering biomarkers of gastroenterological cancer
}

Makoto Suzuki $^{\text {a }}$, Shin Nishiumi ${ }^{\text {a }}$, Atsuki Matsubara ${ }^{\text {a }}$, Takeshi Azuma ${ }^{\text {a }}$, Masaru Yoshida ${ }^{\text {a,b,c* }}$

${ }^{a}$ Division of Gastroenterology, Department of Internal Medicine, Kobe University Graduate

School of Medicine, Kobe, Japan

${ }^{\mathrm{b}}$ The Integrated Center for Mass Spectrometry, Kobe University Graduate School of

Medicine, Kobe, Japan

${ }^{c}$ Division of Metabolomics Research, Department of Internal Medicine related, Kobe

University Graduate School of Medicine, Kobe, Japan

*Corresponding author: Masaru Yoshida M.D., Ph.D.

Division of Metabolomics Research, Division of Gastroenterology, The Integrated Center for Mass Spectrometry, Kobe University Graduate School of Medicine, Research Build. A6-010, 7-5-1, Kusunoki-cho, Chu-o-ku, Kobe, Hyogo 650-0017, JAPAN; Tel: +81-78-382-6305; Fax: +81-78-382-6309; E-mail: myoshida@med.kobe-u.ac.jp 


\section{Abstract}

Improvements in analytical technologies have made it possible to rapidly determine the concentrations of thousands of metabolites in any biological sample, which has resulted in metabolome analysis being applied to various types of research, such as clinical, cell biology, and plant/food science studies. The metabolome represents all of the end products and by-products of the numerous complex metabolic pathways operating in a biological system. Thus, metabolome analysis allows one to survey the global changes in an organism's metabolic profile and gain a holistic understanding of the changes that occur in organisms during various biological processes, e.g., during disease development. In clinical metabolomic studies, there is a strong possibility that differences in the metabolic profiles of human specimens reflect disease-specific states. Recently, metabolome analysis of biofluids, e.g., blood, urine, or saliva, has been increasingly used for biomarker discovery and disease diagnosis. Mass spectrometry-based techniques have been extensively used for metabolome analysis because they exhibit high selectivity and sensitivity during the identification and quantification of metabolites. Here, we describe metabolome analysis using liquid chromatography-mass spectrometry, gas chromatography-mass spectrometry, and capillary electrophoresis-mass spectrometry. Furthermore, the findings of studies that attempted to discover biomarkers of gastroenterological cancer are also outlined. Finally, we discuss metabolome analysis-based disease diagnosis.

\section{Keywords}

Metabolome analysis; Metabolomics; Serum; Cancer; Biomarker; Diagnosis 


\section{Abbreviations}

LC-MS, liquid chromatography-mass spectrometry; GC-MS, gas chromatography-mass spectrometry; CE-MS, capillary electrophoresis-mass spectrometry; NMR, nuclear magnetic resonance; FT-IR, Fourier transform-infrared; TCA, tricarboxylic acid; GLUT, glucose transporter; GLS2, glutaminase 2; m/z, mass-to-charge ratio; EI, electron-impact ionization; TMS, trimethylsilyl; Q, single quadrupole; IT, ion trap; MS/MS, tandem mass spectrometry; MRM, multiple reaction monitoring; QqQ, triple quadrupole; TOF, time-of-flight; FTICR, Fourier transform ion cyclotron resonance; HMDB, the Human Metabolome Database; KEGG, Kyoto Encyclopedia of Genes and Genomes; PCA, principal component analysis; OPLS-DA, orthogonal projection to latent structures-discriminant analysis; MLR, multiple logistic regression analysis; OSCC, oral squamous cell carcinoma; UPLC, ultra performance liquid chromatography; MALDI, matrix-assisted laser desorption/ionization; ROC, receiver operating characteristic; CEA, carcinoembryonic antigen; SFE, supercritical fluid extraction; VOCs, volatile organic compounds; HS-SPME headspace-solid phase microextraction; ITEX, in-tube extraction; QC, quality control; QA, quality assurance 


\section{Introduction}

Comprehensive investigations based on genomics, transcriptomics, proteomics, and metabolomics play an important role in life science. Metabolomics is the study of the final step in the "omics" cascade and is used to acquire comprehensive biological information about low molecular weight metabolites $(<1,000 \mathrm{Da})[1,2]$. The metabolome is the endpoint of the omics cascade, and hence, is the closest point in the cascade to the phenotype; therefore, studies of the metabolome can provide snapshots of the physiological state of an organism (Fig. 1). Over the last decade, metabolome analysis has developed markedly, as demonstrated by the increasing numbers of metabolome analysis-based publications in various research fields, such as clinical, cell biology, and plant/food science studies [3-6]. These developments have mainly been driven by improvements in analytical technologies, which have made it possible to rapidly analyze biological samples for thousands of metabolites. Metabolome analysis involves a qualitative and quantitative approach to analyzing all of the metabolites present in organisms. Currently, two complementary approaches are used for metabolome analysis: metabolic profiling and metabolic fingerprinting. Metabolic profiling describes analyses that focus on a group of metabolites, e.g., a class of compounds, such as carbohydrates, amino acids, or the metabolites associated with a specific pathway [7]. Metabolic profiling requires unambiguous qualitative identification of the metabolites and correction for analytical inaccuracies [8]. Metabolic fingerprinting is a sample classification strategy based on the target samples' spectral patterns, which are considered to be derived from their biological state and/or origin [9]. Many analytical techniques based on liquid chromatography-mass spectrometry (LC-MS), gas chromatography-mass spectrometry (GC-MS), capillary electrophoresis-mass spectrometry (CE-MS), nuclear magnetic resonance (NMR) spectroscopy, or Fourier transform-infrared (FT-IR) spectroscopy have been developed for metabolome analysis [10-14]. Since 
MS-based techniques exhibit high selectivity and sensitivity during the identification and quantification of metabolites, they have been extensively used for metabolic profiling. On the other hand, NMR and FT-IR spectroscopy display low selectivity, but can be used to discriminate between biological samples on the basis of differences in their metabolite profiles. Therefore, these techniques are often used for metabolic fingerprinting.

Recently, metabolome analysis has been increasingly used to examine biofluids, e.g., blood, urine, or saliva, for biomarker discovery and disease diagnosis [15-18]. Most cancers are difficult to detect in their early stages because they exhibit a long asymptomatic period. Moreover, there is currently a lack of highly sensitive and specific cancer diagnostic tools. Since cancer cells are known to display highly unique metabolic phenotypes, metabolome analysis could be used to identify metabolic profiles, fingerprints, or signatures that would be useful for detecting cancer and assessing the pharmacodynamic effects of therapy. In clinical research, its utility has been demonstrated by the identification of new biomarkers for the diagnosis of gastroenterological diseases [19].

Most of the metabolic processes in the body, such as energy metabolism and amino acid catabolism, are common to all living cells. However, some metabolic pathways are up- or down-regulated in cancer cells. Metabolome analysis aims to determine the differences between biological samples based on their metabolic profiles or fingerprints. Cell growth requires a great deal of energy as well as the raw materials for membranes and other cellular components. Most cancer cells predominantly produce energy by glycolysis rather than oxidative phosphorylation via the tricarboxylic acid (TCA) cycle, even in the presence of an adequate oxygen supply: a phenomenon termed "the Warburg effect" [20]. In addition, it is likely that cancer cells exhibit profound changes in their metabolic pathways, such as those for amino acid metabolism and fatty acid oxidation. Such alterations in the metabolomes of cancer cells result in changes in the compositions of biofluids as well as tissues. Thus, 
metabolome analysis could become an ideal tool for cancer diagnosis.

In this review, we describe metabolome analysis using LC-MS, GC-MS, and CE-MS, as well as the findings of studies that attempted to discover biomarkers of gastroenterological cancer. Finally, we discuss metabolome analysis-based disease diagnosis.

\section{The basis of carcinogenesis and metabolic alterations}

Cancers are caused through the multistep accumulation of mutations in both oncogenes and tumor suppressor genes that control the growth, differentiation, and other behaviors of cells $[21,22]$. Evidence has accumulated that the mutations of oncogenes and tumor suppressor genes can stimulate the transcription of a number of genes that encode the proteins mediating the metabolic pathways [23]. Among them, $A P C, K R A S$, and $p 53$ have been reported to play an important role in regulating several aspects of cellular metabolism.

The mutation of the APC gene occurs in the early stages of colorectal and gastric carcinogenesis $[24,25]$. APC proteins negatively regulate the Wnt pathway by aiding the degradation of $\beta$-catenin [26]. The Wnt pathway regulates stem cell pluripotency and cell fate decisions during development. The mutation of $A P C$ gene results in the stabilization of $\beta$-catenin and activation of the Wnt pathway, and then leads to aberrant cellular proliferation. Recently, it was revealed by proteome analysis that $\beta$-catenin activation in mouse liver might affect glutamine and glucose metabolism [27]. In addition, the metabolomic status of cells and tissues with $A P C$ gene mutation indicated that the $A P C$ gene might regulate amino acid related pathways and other energy related metabolic pathways [28].

The mutation of the KRAS gene is an early step in the sequence of pancreatic carcinogenesis. KRAS is a member of the RAS family of GTP-binding proteins that mediate a wide variety of cellular functions including proliferation, differentiation, and survival [29]. The KRAS gene can be activated by a point mutation that permits it to continue to stimulate cell 
proliferation even when the counter regulatory signals are trying to halt the process. Oncogenic KRAS signaling promotes glucose uptake by increasing glucose transporter (GLUT) 1 expression but decreases its utilization in the TCA cycle [30,31].

The p53 tumor suppressor gene is the most commonly mutated gene in human cancer [32-36]. As a transcription factor, the p53 protein mainly exerts its function in tumor suppression through its transcriptional regulation of the target genes to initiate various cellular responses [37]. Recent studies have revealed a novel function of p53 in regulation of cellular metabolism. In cancer cells, mitochondrial oxidative phosphorylation activity is decreased in association with changes in the expression of synthesis of cytochrome c oxidase 2 that is regulated by p53 [38]. The expression of p53 protein also influences glycolysis by regulating the levels of a series of gene products. The mutation of p53 gene abolishes the repressive effect on GLUT1 and GLUT4 that influence glucose metabolism and cell energy supply [39]. The p53 protein can induce the expression of TP53-induced glycolysis and apoptosis regulator, which lowers the intracellular concentration of fructose-2,6-bisphosphate that promotes glycolysis [40]. Additionally, the loss of p53 is associated with the increased level of phosphoglycerate mutase, an enzyme that is part of glycolytic pathway, and thus enhanced glycolysis [41]. Recently, PARK2, a Parkinson disease associated gene, has been suggested to contribute to the functions of p53 in glucose metabolism [42]. Moreover, the status of p53 is a key determinant of the Warburg effect that shows the aberrant conversion of pyruvate into lactate instead of acetyl-CoA in the presence of oxygen. The p53 protein decreases the level of pyruvate dehydrogenase kinase 2, which inactivates acetyl-CoA production by phosphorylating the pyruvate dehydrogenase complex [43].

In addition, p53 regulates many other cellular metabolism such as fatty acid oxidation and glutaminolysis. Lpin1 is induced by p53 in response to glucose starvation, which in turn results in the up-regulation of fatty acid oxidation [44]. The p53 protein increases glutaminase 
2 (GLS2) expression, which enhances the production of glutamate and $\alpha$-ketoglutarate in cells, and thereby promotes mitochondrial oxidative phosphorylation [45]. Furthermore, GLS2 regulates antioxidant defense functions in cells by increasing reduced glutathione levels.

The reprogramming of energy metabolism was recently recognized as an emerging hallmark of cancer [46]. Cellular metabolomics is a useful tool for studying the abnormal metabolism of diseases, and it can provide information about the metabolic alterations and the upstream regulative mechanism in cancers. However, the relationship between cellular metabolism and gastroenterological cancer biomarkers has remained poorly understood yet. Therefore, further studies are required to understand the metabolic alterations in carcinogenesis.

\section{Background of metabolome analysis}

\subsection{Analytical platforms for metabolome analysis}

In metabolomic investigations, it is necessary to comprehensively determine the "global" metabolic profiles of complex samples, such as biofluids and tissue extracts. It is considered that analytical methods based on chromatographic techniques coupled with mass spectrometry are likely to become a major source of global metabolic profiling data due to its high selectivity and sensitivity. Generally, mass spectrometry provides information about the mass-to-charge ratios $(\mathrm{m} / \mathrm{z})$ and fragmentation patterns of compounds. Electrospray ionization and atmospheric pressure chemical ionization, which are used in LC-MS, are soft ionization methods and cause much less compound fragmentation than other ionization methods [47]. In contrast, electron-impact ionization (EI), which is used in GC-MS, gives rise to fragment ions derived from compound cleavage.

GC-MS spectra are highly reproducible, and standard GC-MS spectra databases have already been constructed, e.g., the National Institute of Standards and Technology and the 
Mass Spectral and Retention time Index libraries [48]. As most metabolites are polar and non-volatile in nature, metabolic profiling with GC-MS usually requires chemical derivatization of their polar functional groups to reduce the polarity of the metabolites and increase their thermal stability and volatility. The most commonly used derivatization procedure is oximation with methoxamine hydrochloride followed by trimethylsilyl (TMS) derivatization with $\mathrm{N}$-methyl-trimethylsilyltrifluoroacetamide [49]. Combining GC-MS with derivatization is the preferred method for analyzing the small polar metabolites involved in primary metabolism, such as sugars, organic acids, and amino acids. However, the disadvantage is that some derivatized products are not stable, and can degrade during injection and separation.

LC-MS can be used to analyze a variety of hydrophobic or hydrophilic metabolites depending on the separation column used. Reversed phase liquid chromatography, which is normally performed with $\mathrm{C} 18$ columns, can be used to separate hydrophobic or semi-polar compounds. On the other hand, hydrophilic interaction liquid chromatography can be used to separate polar compounds including sugars, amino sugars, amino acids, vitamins, carboxylic acids, and nucleotides [50]. In addition, an improved LC-MS method, in which ion-pair chromatography and a conventional C18 column were used to analyze anionic metabolites, was recently developed and was found to produce reproducible results [51]. However, the use of gradient elution ion-pair chromatography has been limited due to the need for a long column equilibration time associated with the ion-pair reagent re-distribution. Moreover, the ion-pair reagents can never be removed fully from the LC-MS system even with extensive washing.

CE-MS has recently been used as a powerful tool for the rapid analysis and efficient resolution of ionic metabolites including amino acids, organic acids, nucleotides, and sugar phosphates and does not require any chemical derivatization procedures [52]. Most of the abovementioned metabolites are involved in central metabolic pathways, such as the 
glycolytic pathway and the TCA cycle. CE-MS has several advantages. For example, the sample preparation procedures are simple, rapid, and common to many types of compound, and a small injection sample volume is sufficient (in the order of nanoliters). However, the migration times of each compound are often less reproducible due to the difference of sample matrix and temperature in the environment. Therefore, marker compounds, which can be used to correct the migration times, are applied to each sample for the peak alignment and identification.

Various types of MS instruments can be combined to produce suitable separation techniques, as mentioned above. Due to its relatively low cost, single quadrupole (Q)-MS remains popular. Ion trap (IT)-MS is also employed extensively as it enables tandem mass spectrometry (MS/MS) to be performed, although it has a narrow dynamic analysis range. Metabolome analysis using MS/MS is applied to obtain structural information based on the fragmentation patterns of metabolites. In the case of targeted LC-MS analysis, multiple reaction monitoring $(\mathrm{MRM})$ using triple quadrupole (QqQ)-MS enables detection with high sensitivity, selectivity, reproducibility, and a broad dynamic analysis range. Furthermore, widely targeted metabolome analysis, which is high-throughput and high-sensitive analysis of broadly targeted metabolites, with QqQ-MS makes it easy to detect metabolite peaks and accurately quantify metabolite levels. Widely targeted metabolome analysis is an alternative to non-targeted metabolome analysis [53,54]. In addition, Tsugawa et al. have developed a practical GC-QqQ-MS system for the highly sensitive and selective metabolic profiling of TMS-derivatized compounds using the EI method [55].

The complexity of biological samples can mean that peaks with similar nominal masses cannot be identified correctly. For non-targeted metabolome analysis, high resolution and highly accurate mass techniques including time-of-flight (TOF)-MS, Fourier transform ion cyclotron resonance (FTICR)-MS, or Orbitrap-MS allow the accurate identification of known 
metabolites and the potential identification of unknown compounds [56-58].

The major obstacle in metabolome analysis is that the MS signal intensity is dependent not only on metabolite concentration, but also on its chemical structure and sample matrix. Ion suppression and enhancement caused by matrix effects result in inaccurate quantification of the metabolites [59]. As the solution for absolute quantification, stable isotopically labeled standards with almost equal chemical properties are used as internal standards, which account for sample processing variation and matrix effects encountered during MS analysis.

These technical advances in analytical platforms and methodologies have accelerated biomarker discovery with metabolomic approaches. Recent reports about the discovery of biomarkers of gastroenterological cancer using metabolome analysis are summarized in Table 1.

\subsection{Data analysis and statistical approaches}

In biomarker discovery and basic metabolomic studies, metabolic profiling requires a data preprocessing step to determine the biological origins of specific metabolites. It is necessary to convert the large amounts of data generated by metabolome analysis into $\mathrm{m} / \mathrm{z}$, retention time, and signal intensity information for each metabolite peak. Fortunately, automatic MS data preprocessing, alignment, and identification software for metabolome analysis is freely available, e.g., MetAlign, XCMS, MZmine, AIoutput, and MRMPROBS (Table 2) [60-64].

Public metabolite databases that include detailed mass spectrometric, physicochemical, clinical, and molecular biology/biochemistry data are used for the subsequent metabolite identification step. For metabolome analysis, metabolite peak identification can be performed using in-house library including retention time and MS data of authentic standard compounds. If it is not possible, analytes are provisionally identified using MS/MS fragment ion data by referencing major metabolite databases such as METLIN and MassBank [65,66]. METLIN is 
a repository of metabolite information to assist metabolite identification by providing MS data containing over 64,000 structures. MassBank is designed for public sharing of reference MS data, which containing over 40,000 spectra analyzed under non-standardized experimental conditions. The Human Metabolome Database (HMDB) and Kyoto Encyclopedia of Genes and Genomes (KEGG) are particularly useful for biological interpretation in studies of the human metabolome [67,68]. HMDB includes over 41,000 compounds that humans ingest, metabolize, catabolize, or come into contact with. It has detailed information about physicochemical properties, spectral data, biological properties, concentrations (normal and abnormal), associated disorders, enzymes, and transporters. KEGG is widely used database containing metabolic pathways from a wide variety of organisms. These pathways are linked with metabolite and protein/enzyme information. Although, each one of these databases covers only a fraction of the metabolome, integration of the search results from these databases is expected to yield a more comprehensive coverage.

Metabolic profiling datasets can be statistically analyzed by chemometric approaches, including principal component analysis (PCA) and orthogonal projection to latent structures-discriminant analysis (OPLS-DA) as a means of evaluating biological changes $[69,70]$. Both of the techniques are used for reduction of metabolic profiling data consisting of a large number of compounds. PCA has been extensively applied to data mining and pattern recognition for unsupervised dimensionality reduction. Unsupervised approaches do not require any a priori knowledge about the samples for classification. Despite its popularity, PCA suffers from a lack of interpretability because the reduced dimensions are linear combinations of a large number of original features. In OPLS-DA, where the prediction value for classification is available, it would be more helpful to incorporate class information into the mapping and derive a supervised projection for the original features. However, OPLS-DA suffers from overfitting, meaning that the model classifies the training data well, but the 
validation data are classified poorly. In order to avoid overfitting, it is necessary to use additional techniques, e.g., cross-validation and permutation test. When sample groups are clearly separated, the metabolites that are most responsible for the separation can be determined by loadings plots derived from PCA or OPLS-DA. In addition, recent clinical studies employed multiple logistic regression analysis (MLR) and identified important metabolomic features for cancer diagnosis [71-74].

\section{Biomarker discovery using metabolome analysis}

The clinical diagnosis of gastroenterological cancer is still a major challenge for physicians and oncologists. Gastroenterological cancer, which refers to a group of cancers that affect the organs of the gastrointestinal tract, including oral, esophageal, gastric, colorectal, hepatic, and pancreatic cancer, remains relatively asymptomatic until the progressive state, and its prognosis is poor. Metabolome analysis has been used to identify potential biomarkers and therapeutic targets in a variety of diseases, and the recent reports about the use of metabolome analysis in patients with gastroenterological cancer are summarized in Table 1. In these studies, low molecular weight metabolites were extracted from human specimens, such as serum, plasma, saliva, urine, feces, mucosal tissue, and other tissue samples, and then examined using metabolomic approaches. In our previous study, we performed a GC-MS-based metabolome analysis of gastroenterological cancer and discovered several biomarker candidates. Below, we describe a few examples of the application of metabolome analysis to biomarker discovery, focusing on our results.

\subsection{Oral cancer}

Over $90 \%$ of oral cancer cases involve oral squamous cell carcinoma (OSCC), which arises from the oral mucosal lining [75]. In OSCC, if the cancer is detected at the T1 stage (T 
stage represents the size of the tumor and the extent of its invasion), the five-year survival rate is over $80 \%$ [76]. On the other hand, if the cancer is diagnosed at an advanced stage (T3 or T4), the five-year survival rate ranges from 20 to $40 \%$. Therefore, it is important to detect OSCC at an early stage. The detection of conventional tumor markers, e.g., SCC antigen; diagnostic imaging techniques, such as computed tomography; and biopsy are not suitable for OSCC screening because conventional tumor markers have low sensitivity for the disease, diagnostic imaging is expensive, and biopsy is invasive.

Many studies of OSCC biomarkers have been performed by various research groups. Wei et al. reported the salivary metabolite signatures for a group of 37 OSCC patients and 34 healthy volunteers using ultra performance liquid chromatography (UPLC)-Q-TOF/MS to evaluate the detection of OSCC [77]. Xie et al. identified distinct urinary metabolic signatures using OSCC ( $n=34)$ and healthy controls $(n=34)$ for diagnosis of early stage of OSCC with GC-MS [78]. Additionally, Maurer et al. demonstrated that peptide fingerprints, which is obtained from brush biopsy samples by using matrix-assisted laser desorption/ionization (MALDI)-TOF MS, could discriminate between OSCC samples $(\mathrm{n}=26)$ and non-cancer controls $(n=26)[79]$.

In our previous study, we performed a serum metabolome analysis of OSCC using a GC-MS-based method [80]. In that study, we used 18 OSCC patients and 18 healthy volunteers as a training set, and an OSCC prediction model was established via MLR. Then, the validity of the predictive model was assessed using a validation set consisting of OSCC patients $(n=17)$ and healthy volunteers $(\mathrm{n}=17)$. As a result, glycerate, serine, laurate, $N$-acetyl-L-aspartate, asparagine, ornithine, and heptadecanoate were selected as biomarker candidates because significant and similar alterations in their serum levels were observed in both the training and validation sets. In particular, a diagnostic system for OSCC based on combining several metabolite biomarker candidates exhibited high sensitivity and specificity in receiver operating 


\subsection{Esophageal and gastric cancer}

Esophageal cancer is the sixth most common cause of cancer death worldwide [81]. The number of esophageal adenocarcinoma cases has been slowly increasing and now accounts for approximately $70 \%$ of all esophageal cancer patients in Europe and America [82]. Gastric cancer is the fourth most common type of cancer and the second most frequent cause of cancer death [83]. Upper gastrointestinal endoscopy and barium esophagrams are used for early screening for esophageal and gastric cancer, but these methods are invasive, uncomfortable, and costly. Furthermore, serological marker tests have limitations, and the detection of esophageal cancer depends on performing adequate biopsies of the esophageal mucosa, although the accuracy of tissue biopsies involving endoscopically abnormal mucosal tissue ranges from 25 to $60 \%$ [84].

Many researchers have attempted to find novel biomarkers of esophageal and gastric cancer using various methods including proteomic and transcriptomic approaches. In esophageal squamous cell carcinoma, SCC, p53, cytokeratins, myomegalin, tripartite motif-containing protein 21 , and peroxiredoxin VI protein have been identified as novel markers [85-87]. In gastric cancer, four specific protein markers; i.e., heat shock protein 27, 


\subsection{Colorectal cancer}

Recently, the number of patients with colorectal cancer has been increasing due to lifestyle changes. Colorectal cancer is one of the most common causes of cancer death in many developed countries [91]. The present diagnostic and screening methods for colorectal cancer, such as the fecal occult blood test; fecal-based DNA tests; and tests for serum based protein markers, e.g., carcinoembryonic antigen (CEA), have certain limitations. Endoscopic 
examination of the colon remains the gold standard for diagnosis, although it is invasive and unsuitable for reducing the risk of morbidity and mortality. Therefore, it is necessary to establish new screening methods for the early diagnosis of colorectal cancer that are highly sensitive, specific, easy to perform, and non-invasive.

Qiu et al. utilized GC-MS-based urinary metabolic profiling and detected alterations in the concentrations of 16 metabolites in urine samples collected from a total of 60 colorectal cancer patients and 63 healthy volunteers [92]. Among them, the alterations in the levels of succinate, phenylacetylglutamine, 2-hydroxyhippurate, and 5-hydroxytryptophan were monitored in the same patients after surgery. In addition, they used the same method to analyze urine samples from Sprague-Dawley rats with 1,2-dimethylhydrazine-induced precancerous colorectal lesions $(n=8)$ and healthy control group $(n=8)$. Then, the metabolic alterations that occurred in the colorectal cancer patients were compared and verified with those that occurred in the chemically-induced colorectal lesion rat model. Significantly increased tryptophan metabolism, TCA cycle disturbance, and gut microflora metabolism were observed in both the colorectal cancer patients and the rat model. The down-regulation of intermediates in the TCA cycle is consistent with the Warburg effect, whereas further studies are required to clarify the mechanism behind the other metabolic alterations.

In a serum-based clinical study, we investigated a new screening method for the early diagnosis of colorectal cancer based on GC-MS metabolomics [73]. Serum metabolite levels were compared between colorectal cancer patients $(n=60 ; n=12$ for each stage from 0 to 4$)$ and age- and sex-matched healthy volunteers $(n=60)$ as a training set. The metabolites whose levels displayed significant changes were subjected to MLR with the stepwise variable selection method, and a colorectal cancer prediction model was established. The established prediction model was composed of 4 metabolites; i.e., 2-hydroxybutyrate, aspartate, kynurenine, and cystamine (cysteamine+cystamine). In ROC analysis, the predictive model 


\subsection{Pancreatic cancer}

Worldwide, more than 200,000 people develop pancreatic cancer annually, and $98 \%$ of these patients die of the disease [83]. The high mortality rate of pancreatic cancer is due to the lack of adequate systemic therapies and the high frequency of metastasis at the time of diagnosis. The clinical symptoms of pancreatic cancer are usually unremarkable until the cancer has progressed to an advanced stage. Furthermore, there is no diagnostic method for its early detection. Many researchers have attempted to find novel biomarkers for pancreatic cancer using proteomic approaches. Hibi et al. reported the proteomic analysis of 5 human pancreatic cancer cell lines, which show the difference of perineural invasion, and then provided in vivo evidence that synuclein- $\gamma$ is closely involved in perineural invasion/distant metastasis and is a significant prognostic factor in pancreatic cancer [94]. Sitek et al. reported 
the quantitative proteome study to investigate protein changes between normal ductal cells, pancreatic intraepithelial neoplasia lesions, and carcinoma cells [95]. In that study, major vault protein, anterior gradient 2, 14-3-3 sigma, annexin A4, and S100A10 were identified as biomarker candidates, and then validated by immunohistochemistry using tissue specimen from patients suffering from pancreatic ductal adenocarcinoma and pancreatitis. Yu et al. employed two-dimensional LC-MS/MS analysis coupled with a stable isotope labeled proteome standard for pancreatic cancer biomarker discovery [96]. A total of 1065 proteins were identified, of which 121 proteins were present at 1.5 -fold or greater concentrations in the sera of patients with pancreatic cancer. Validation of these findings was successfully performed using enzyme-linked immunosorbent assays for two proteins, intercellular adhesion molecule 1 and basal cell adhesion molecule. Nevertheless, these candidate proteins need further to be evaluated in clinical settings.

Sugimoto et al. compared the comprehensive salivary metabolic profiles of patients with oral $(n=69)$, breast $(n=30)$, or pancreatic cancer $(n=18)$; patients with periodontal disease $(n$ $=11)$; and healthy controls $(\mathrm{n}=87)$ [71]. Saliva is composed of $99 \%$ water combined with minerals; mucus; electrolytes; nucleic acids; and proteins, such as enzymes, enzyme inhibitors, growth factors, cytokines, immunoglobulins, and other glycoproteins [97]. Since saliva is a filtrate of blood, it reflects the physiological state of the body and so could be used to determine an individual's clinical status for disease diagnosis. Using CE-MS, Sugimoto et al. identified individual cancer-specific markers that exhibited high discriminative ability, thereby demonstrating the utility of salivary metabolomics for cancer diagnosis.

We have found that it is possible to discriminate between patients with pancreatic cancer $(\mathrm{n}=20)$ and healthy volunteers $(\mathrm{n}=9)$ using GC-MS-based metabolome analysis of human serum samples [98]. Similar results were obtained for the NMR-based metabolic profiling of rat tissues [99] and NMR-based serum metabolome analysis in humans [100]. Furthermore, we 
constructed a diagnostic model for pancreatic cancer using GC-MS-based human serum metabolome analysis and then confirmed its diagnostic performance via validation analysis and comparisons with conventional tumor markers [74]. Our GC-MS method mainly targeted water-soluble metabolites, and a total of 159 compounds were detected in the subjects' sera. After excluding contaminants or unstable compounds with relative standard deviation values over 20\%, 45 metabolites were subjected to semiquantitative evaluation via statistical approaches. For the semiquantitative evaluation, the peak height of each metabolite was calculated and normalized to the peak height of 2-isopropylmalate as an internal standard. Then, 4 metabolites were selected from the 45 metabolites via the stepwise method; that is, xylitol, 1,5-anhydro-D-glucitol, histidine, and inositol. Using these metabolites, a diagnostic model for pancreatic cancer was established using MLR. In the training set study, which included 43 pancreatic cancer patients and 42 healthy volunteers, the model was found to possess high sensitivity (86.0\%) and specificity (88.1\%) in ROC analysis. The utility of the model was compared to those of CA19-9 and CEA in the validation set study, which included 42 pancreatic cancer patients, 41 healthy volunteers, and 23 chronic pancreatitis patients. The model displayed high sensitivity $(71.4 \%)$ for detecting pancreatic cancer. On the contrary, the sensitivity of CA19-9 was $69.0 \%$ and that of CEA was $35.7 \%$. Furthermore, the sensitivities of the model, CA19-9, and CEA in resectable pancreatic cancer were $77.8 \%, 55.6 \%$, and $44.4 \%$, respectively. In the case of chronic pancreatitis, their false positive rates were $17.4 \%$, $30.4 \%$, and $43.5 \%$, respectively. Therefore, it is a promising method for improving the prognosis of pancreatic cancer via its early detection and accurate discrimination from chronic pancreatitis.

\section{Conclusions and perspectives}

So far, a large number of clinical studies using metabolome analysis have been performed to discover disease-specific metabolites. A large number of metabolome analysis 
have been performed to discover disease-specific metabolites. An outline of the experimental process required to produce metabolomic clinical screening tools is shown in Fig. 2. In small-scale screening, some biomarker candidates might produce false positives due to confounding factors, such as the effects of diet. For example, among the serum metabolites detected by our GC-MS-based metabolome analysis, the tryptophan concentrations observed in the afternoon and at night were significantly decreased in comparison with those detected in the morning [73]. In other studies, some amino acids were reported to show intra- and inter-day variations $[101,102]$. Researchers might also encounter confounding factors related to differences in the sample collection and sample handling methods, such as the timing of the participants' mealtimes, the collection method, and/or the time until freezing. Therefore, the enforcement of consistent sample collection and storage protocols is important for studies based on metabolome analysis. Moreover, the levels of detected metabolites exhibit intra- and inter-day variance and can be affected by instrument instability. Thus, it is also necessary to subject metabolomic data to careful validation.

In addition, the extraction of metabolites and their subsequent pretreatment for mass spectrometric analysis must be also considered when evaluating the practical utility of metabolome analysis. At present, metabolites are basically extracted via liquid-liquid and solid-phase extraction, but easier extraction and pretreatment methods are required to make metabolome analysis more practical. Therefore, an automated analysis system that performs all of the required processes from metabolite extraction to mass spectrometric analysis is expected to be developed. To this end, the usage of dried blood spot sampling, in which blood is blotted and dried on filter paper, and supercritical fluid extraction (SFE), which is an extraction technique using supercritical carbon dioxide, might be useful. In particular, SFE does not require sample pretreatment and is mainly used for extracting hydrophobic compounds. Recently, SFE was combined with mass spectrometry, and the analysis of blood 
metabolites using this method accompanied by dried plasma spotting is currently being investigated [103].

Volatile organic compounds (VOCs) profiling is a novel research area, where the composition of the VOCs emitted by the biological samples can be correlated to the physiological state. VOCs in human specimens include the metabolites such as alcohols, aldehydes, furans, ketones, pyrroles, terpenes, and other heterocyclic compounds. VOCs profiling is much more difficult because of their volatility, structural diversity, and differences in their polarity and concentrations. Recently, headspace-solid phase microextraction (HS-SPME) with GC-MS has been used for the identification of volatile disease biomarkers [104-106]. This technique does not require any solvent or concentration step for volatile analysis. Furthermore, Kakuta et al. employed in-tube extraction (ITEX), which provides lower fragility and longer extraction phase lifetimes as well as lower detection limit compared to those of SPME, and demonstrated metabolic profiling of oxidized lipid derived volatiles in blood [107]. However, the current lack of consensus regarding analytical techniques still hampers clinical usefulness of volatile biomarkers.

Multi-platform metabolome analysis is expected to enable the expansion of metabolite detection, leading to efficient biomarker screening. Protocols for large-scale metabolomic studies involving LC-MS and GC-MS have been developed by the Human Serum Metabolome Consortium (http://www.husermet.org/index.php). As the frequency of large-scale metabolic profiling increases, the need for data quality control (QC) and quality assurance (QA) has become much more apparent [108,109]. QC samples, with a similar metabolic and matrix composition to those of biological samples, should be periodically analyzed throughout an analytical run in order to check the reproducibility of the data produced by the instruments used and provide robust QA for each metabolite peak. Subsequently, the biological validation of candidate biomarkers in in vivo animal 
experiments or in vitro cell culture experiments is important. In addition, knowledge-based pathway analysis allows one to analyze metabolomic data in the context of the relevant metabolic pathways and to understand biological processes and phenotypes in diseases related to the candidate biomarkers [110]. Metabolome analysis has great potential to be an effective tool for the early diagnosis of a variety of diseases. Further developments in the area of advanced metabolite detection technologies and data analysis will aid the widespread usage of metabolome analysis in clinical studies.

\section{Acknowledgements}

This study was supported in part by a grant from the 'Young Researchers Training Program for Promoting Innovation' run by the Special Coordination Fund for Promoting Science and Technology from the Ministry of Education, Culture, Sports, Science, and Technology of Japan [A.M., T.A.]; a Grant-in-Aid for Scientific Research (B) from the Ministry of Education, Culture, Sports, Science, and Technology of Japan [M.Y.];

Grant-in-Aid for Exploratory Research from the Ministry of Education, Culture, Sports, Science, and Technology of Japan [M.Y.]; and a grant from the Development of Systems and Technology for Advanced Measurement and Analysis Project (JST) [M.S., M.Y.].

\section{References}

[1] S. Oliver, Nature 403 (2000) 601.

[2] O. Fiehn. Plant Mol. Biol. 48 (2002) 155.

[3] J.L. Spratlin, N.J. Serkova, S.G. Eckhardt, Clin. Cancer Res. 15 (2009) 431.

[4] M.R. Mashego, K. Rumbold, M. De Mey, E. Vandamme, W. Soetaert, J.J. Heijnen, Biotechnol. Lett. 29 (2007) 1.

[5] D.S. Wishart, Trends Food Sci. Technol. 19 (2008) 482.

[6] K. Saito, Curr. Opin. Plant Biol. 16 (2013) 373.

[7] T. Niwa, J. Chromatogr. B 379 (1986) 313. 
[8] M. Oldiges, S. Lütz, S. Pflug, K. Schroer, N. Stein, C. Wiendahl, Appl. Microbiol. Biotechnol. 76 (2007) 495.

[9] O. Fiehn, Compar. Funct. Genom. 2 (2001) 155.

[10] G. Theodoridis, H.G. Gika, I.D. Wilson, Trac-Trends Anal. Chem. 27 (2008) 251.

[11] K.K. Pasikanti, P.C. Ho, E.C.Y. Chan, J. Chromatogr. B 871 (2008) 202.

[12] T. Soga, Y. Ohashi, Y. Ueno, H. Naraoka, M. Tomita, T. Nishioka, J. Proteome Res. 2 (2003) 488.

[13] J.K. Nicholson, J.C. Lindon, E. Holmes, Xenobiotica 29 (1999) 1181.

[14] D.I. Ellis, R. Goodacre, Analyst 131 (2006) 875.

[15] A. Zhang, H. Sun, X. Wang, Anal. Bioanal. Chem. 404 (2012) 1239.

[16] A. Zhang, H. Sun, X. Wu, X. Wang, Clin. Chim. Acta. 414 (2012) 65.

[17] N. Spielmann, D.T. Wong, Oral Dis. 17 (2011) 345.

[18] D.J.Y. Ng, K.K. Pasikanti, E.C.Y. Chan, Metabolomics 7 (2010) 155.

[19] M. Yoshida, N. Hatano, S. Nishiumi, Y. Irino, Y. Izumi, T. Takenawa, T. Azuma, J. Gastroenterol. 47 (2012) 9.

[20] O. Warburg, Science 123 (1956) 309.

[21] C.R. Boland, Gastrointest. Endosc. Clin. N. Am. 18 (2008) 401.

[22] S.D. Markowitz, M.M. Bertagnolli, N. Engl. J. Med. 361 (2009) 2449.

[23] A.J. Levine, A.M. Puzio-Kuter, Science 330 (2010) 1340.

[24] S.M. Powell, N. Zilz, Y. Beazer-Barclay, T.M. Bryan, S.R. Hamilton, S.N. Thibodeau, B. Vogelstein, K.W. Kinzler, Nature 359 (1992) 235.

[25] G. Tamura, C. Maesawa, Y. Suzuki, H. Tamada, M. Satoh, S. Ogasawara, M. Kashiwaba, R. Satodate, Cancer Res. 54 (1994) 1149.

[26] B.M. Gumbiner, Curr. Opin. Cell Biol. 7 (1995) 634.

[27] P. Chafey, L. Finzi, R. Boisgard, M. Caüzac, G. Clary, C. Broussard, J.P. Pégorier, F. Guillonneau, P. Mayeux, L. Camoin, B. Tavitian, S. Colnot, C. Perret, Proteomics 9 (2009) 3889.

[28] T Yoshie, S. Nishiumi, Y. Izumi, A. Sakai, J. Inoue, T. Azuma, M. Yoshida, Cancer Sci. 103 (2012) 1010.

[29] S.L. Campbell, R. Khosravi-Far, K.L. Rossman, G.J. Clark, C.J. Der. Oncogene 17 (1998) 1395.

[30] J. Yun, C. Rago, I. Cheong, R. Pagliarini, P. Angenendt, H. Rajagopalan, K. Schmidt, J.K. Willson, S. Markowitz, S. Zhou, L.A. Diaz, V.E. Velculescu, C. Lengauer, K.W. Kinzler, B. Vogelstein, N. Papadopoulos, Science 325 (2009) 1555. 
[31] D. Gaglio, C.M. Metallo, P.A. Gameiro, K. Hiller, L.S. Danna, C. Balestrieri, L. Alberghina, G. Stephanopoulos, F. Chiaradonna, Mol. Syst. Biol. 7 (2011) 523.

[32] M.C. Hollstein, R.A. Metcalf, J.A. Welsh, R. Montesano, C.C. Harris, Proc. Natl. Acad. Sci. U. S. A. 87 (1990) 9958.

[33] C.M. Fenoglio-Preiser, J. Wang, G.N. Stemmermann, A. Noffsinger, Hum. Mutat. 21 (2003) 258.

[34] N.R. Rodrigues, A. Rowan, M.E. Smith, I.B. Kerr, W.F. Bodmer, J.V. Gannon, D.P. Lane, Proc. Natl. Acad. Sci. U. S. A. 87 (1990) 7555.

[35] C.M. Barton, S.L. Staddon, C.M. Hughes, P.A. Hall, C. O'Sullivan, G. Klöppel, B. Theis, R.C.G. Russell, J. Neoptolemos, R.C.N. Williamson, D.P. Lane, N.R. Lemoinel, Br. J. Cancer 64 (1991) 1076.

[36] S.P. Hussain, J. Schwank, F. Staib, X.W. Wang, C.C. Harris, Oncogene 26 (2007) 2166.

[37] A.J. Levine, Cell 88 (1997) 323.

[38] S. Matoba, J.G. Kang, W.D. Patino, A. Wragg, M. Boehm, O. Gavrilova, P.J. Hurley, F. Bunz, P.M. Hwang, Science 312 (2006) 1650.

[39] F. Schwartzenberg-Bar-Yoseph, M. Armoni, E. Karnieli, Cancer Res. 64 (2004) 2627.

[40] K. Bensaad, A. Tsuruta, M.A. Selak, M.N. Vidal, K. Nakano, R. Bartrons, E. Gottlieb, K.H. Vousden, Cell 126 (2006) 107.

[41] H. Kondoh, M.E. Lleonart, J. Gil, J. Wang, P. Degan, G. Peters, D. Martinez, A. Carnero, D. Beach, Cancer Res. 65 (2005) 177.

[42] C. Zhang, M. Lin, R. Wu, X. Wang, B. Yang, A.J. Levine, W. Hu, Z. Feng, Proc. Natl. Acad. Sci. U. S. A. 108 (2011) 16259.

[43] T. Contractor, C.R. Harris, Cancer Res. 72 (2012) 560.

[44] W. Assaily, D.A. Rubinger, K. Wheaton, Y. Lin, W. Ma, W. Xuan, L. Brown-Endres, K. Tsuchihara, T.W. Mak, S. Benchimol, Mol. Cell 44 (2011) 491.

[45] W. Hu, C. Zhang, R. Wu, Y. Sun, A. Levine, Z. Feng, Proc. Natl. Acad. Sci. U. S. A. 107 (2010) 7455.

[46] D. Hanahan, R.A. Weinberg, Cell 144 (2011) 646.

[47] J.B. Fenn, M. Mann, C.K. Meng, S.F. Wong, C.M. Whitehouse, Science 246 (1989) 64.

[48] N. Schauer, D. Steinhauser, S. Strelkov, D. Schomburg, G. Allison, T. Moritz, K. Lundgren, U. Roessner-Tunali, M.G. Forbes, L. Willmitzer, A.R. Fernie, J. Kopka, FEBS Lett. 579 (2005) 1332. 
[49] J. Lisec, N. Schauer, J. Kopka, L. Willmitzer, A.R. Fernie, Nat. Protoc. 1 (2006) 387.

[50] S. Cubbon, C. Antonio, J. Wilson, J. Thomas-Oates, Mass Spectrom. Rev. 29 (2010) 671.

[51] B. Luo, K. Groenke, R. Takors, C. Wandrey, M. Oldiges, J. Chromatogr. A 1147 (2007) 153.

[52] M.R.N. Monton, T. Soga, J. Chromatogr. A 1168 (2007) 237.

[53] Y. Sawada, K. Akiyama, A. Sakata, A. Kuwahara, H. Otsuki, T. Sakurai, K. Saito, M.Y. Hirai, Plant Cell Physiol. 50 (2009) 37.

[54] H. Kato, Y. Izumi, T. Hasunuma, F. Matsuda, A. Kondo, J. Biosci. Bioeng. 113 (2012) 665 .

[55] H. Tsugawa, Y. Tsujimoto, K. Sugitate, N. Sakui, S. Nishiumi, T. Bamba, E. Fukusaki, J. Biosci. Bioeng. 117 (2014) 122.

[56] W.C. Wiley, I.H. McLaren, Rev. Sci. Instrum. 26 (1955) 1150.

[57] J. Han, R.M. Danell, J.R. Patel, D.R. Gumerov, C.O. Scarlett, J.P. Speir, C.E. Parker, I. Rusyn, S. Zeisel, C.H. Borchers, Metabolomics 4 (2008) 128.

[58] Q. Hu, R.J. Noll, H. Li, A. Makarov, M. Hardman, R. Graham Cooks, J. Mass Spectrom. 40 (2005) 430.

[59] T.M. Annesley, Clin. Chem. 49 (2003) 1041.

[60] A. Lommen, Anal. Chem. 81 (2009) 3079.

[61] C.A. Smith, E.J. Want, G. O’Maille, R. Abagyan, G. Siuzdak, Anal. Chem. 78 (2006) 779 .

[62] T. Pluskal, S. Castillo, A. Villar-Briones, M. Oresic, BMC Bioinformatics 11 (2010) 395.

[63] H. Tsugawa, Y. Tsujimoto, M. Arita, T. Bamba, E. Fukusaki, BMC Bioinformatics 12 (2011) 131.

[64] H. Tsugawa, M. Arita, M. Kanazawa, A. Ogiwara, T. Bamba, E. Fukusaki, Anal. Chem. 85 (2013) 5191.

[65] C.A. Smith, G. O’Maille, E.J. Want, C. Qin, S.A. Trauger, T.R. Brandon, D.E. Custodio, R. Abagyan, G. Siuzdak, Ther. Drug Monit. 27 (2005) 747.

[66] H. Horai, M. Arita, S. Kanaya, Y. Nihei, T. Ikeda, K. Suwa, Y. Ojima, K. Tanaka, S. Tanaka, K. Aoshima, Y. Oda, Y. Kakazu, M. Kusano, T. Tohge, F. Matsuda, Y. Sawada, M.Y. Hirai, H. Nakanishi, K. Ikeda, N. Akimoto, T. Maoka, H. Takahashi, T. Ara, N. Sakurai, H. Suzuki, D. Shibata, S. Neumann, T. Iida, K. Tanaka, K. Funatsu, F. 
Matsuura, T. Soga, R. Taguchi, K. Saito, T. Nishioka, J. Mass Spectrom. 45 (2010) 703.

[67] D.S. Wishart, T. Jewison, A.C. Guo, M. Wilson, C. Knox, Y. Liu, Y. Djoumbou, R. Mandal, F. Aziat, E. Dong, S. Bouatra, I. Sinelnikov, D. Arndt, J. Xia, P. Liu, F. Yallou, T. Bjorndahl, R. Perez-Pineiro, R. Eisner, F. Allen, V. Neveu, R. Greiner, A. Scalbert, Nucleic Acids Res. 41 (2013) D801.

[68] M. Kanehisa, S. Goto, Nucleic Acids Res. 28 (2000) 27.

[69] S. Wold, K. Esbensen, P. Geladi, Chemometrics Intell. Lab. Syst. 2 (1987) 37.

[70] M. Bylesjö, M. Rantalainen, O. Cloarec, J.K. Nicholson, E. Holmes, J. Trygg, J. Chemometr. 20 (2006) 341.

[71] M. Sugimoto, D.T. Wong, A. Hirayama, T. Soga, M. Tomita, Metabolomics 6 (2010) 78.

[72] T. Soga, M. Sugimoto, M. Honma, M. Mori, K. Igarashi, K. Kashikura, S. Ikeda, A. Hirayama, T. Yamamoto, H. Yoshida, M. Otsuka, S. Tsuji, Y. Yatomi, T. Sakuragawa, H. Watanabe, K. Nihei, T. Saito, S. Kawata, H. Suzuki, M. Tomita, M. Suematsu, J. Hepatol. 55 (2011) 896.

[73] S. Nishiumi, T. Kobayashi, A. Ikeda, T. Yoshie, M. Kibi, Y. Izumi, T. Okuno, N. Hayashi, S. Kawano, T. Takenawa, T. Azuma, M. Yoshida, PLoS One 7 (2012) e40459.

[74] T. Kobayashi, S. Nishiumi, A. Ikeda, T. Yoshie, A. Sakai, A. Matsubara, Y. Izumi, H. Tsumura, M. Tsuda, H. Nishisaki, N. Hayashi, S. Kawano, Y. Fujiwara, H. Minami, T. Takenawa, T. Azuma, M. Yoshida, Cancer Epidemiol. Biomarkers Prev. 22 (2013) 571.

[75] C. Scully, D.H. Felix, Br. Dent. J. 199 (2005) 565.

[76] D.T. Wong, Expert Rev. Mol. Diagn. 6 (2006) 267.

[77] J. Wei, G. Xie, Z. Zhou, P. Shi, Y. Qiu, X. Zheng, T. Chen, M. Su, A. Zhao, W. Jia, Int. J. Cancer 129 (2011) 2207.

[78] G.X. Xie, T.L. Chen, Y.P. Qiu, P. Shi, X.J. Zheng, M.M. Su, A.H. Zhao, Z.T. Zhou, W. Jia, Metabolomics 8 (2011) 220.

[79] K. Maurer, K. Eschrich, W. Schellenberger, J. Bertolini, S. Rupf, T.W. Remmerbach, Oral Oncol. 49 (2013) 152.

[80] A. Kimoto, S. Nishiumi, T. Kobayashi, Y. Terashima, H. Suzuki, J. Takeuchi, T. Azuma, T. Komori, M. Yoshida, Head Neck Oncol. 5 (2013) 40.

[81] K. Isono, T. Ochiai, K. Okuyama, S. Onoda, Jpn. J. Surg. 20 (1990) 151. 
[82] Y.K. Yee, B.C.Y. Wong, J. Gastroenterol. Hepatol. 23 (2008) 1627.

[83] A. Jemal, F. Bray, M.M. Center, J. Ferlay, E. Ward, D. Forman, CA-Cancer J. Clin. 61 (2011) 69 .

[84] M. Groome, J. Lindsay, P.E. Ross, J.P. Cotton, T.R. Hupp, J.F. Dillon, Eur. J. Gastroenterol. Hepatol. 20 (2008) 961.

[85] A.S. Bergqvist, M. Bergqvist, D. Brattstrom, P. Hesselius, A. Larsson, O. Brodin, G. Wagenius, Anticancer Res. 21 (2001) 4141.

[86] R.B. Veale, A.L. Thornley, E. Scott, A. Antoni, I. Segal, Br. J. Cancer 58 (1988) 767.

[87] H. Shimada, M. Kuboshima, T. Shiratori, Y. Nabeya, A. Takeuchi, H. Takagi, F. Nomura, M. Takiguchi, T. Ochiai, T. Hiwasa, Int. J. Oncol. 30 (2007) 97.

[88] Y. Fujita, T. Nakanishi, Y. Miyamoto, M. Hiramatsu, H. Mabuchi, A. Miyamoto, A. Shimizu, T. Takubo, N. Tanigawa, Cancer Lett. 263 (2008) 280.

[89] V.W. Davis, D.E. Schiller, D. Eurich, M.B. Sawyer, World J. Surg. Oncol. 10 (2012) 271.

[90] A. Ikeda, S. Nishiumi, M. Shinohara, T. Yoshie, N. Hatano, T. Okuno, T. Bamba, E. Fukusaki, T. Takenawa, T. Azuma, M. Yoshida, Biomed. Chromatogr. 26 (2012) 548.

[91] R. Siegel, D. Naishadham, A. Jemal, CA-Cancer J. Clin. 62 (2012) 10.

[92] Y. Qiu, G. Cai, M. Su, T. Chen, Y. Liu, Y. Xu, Y. Ni, A. Zhao, S. Cai, L.X. Xu, W. Jia, J. Proteome Res. 9 (2010) 1627.

[93] S.A. Ritchie, P.W.K. Ahiahonu, D. Jayasinghe, D. Heath, J. Liu, Y. Lu, W. Jin, A. Kavianpour, Y. Yamazaki, A.M. Khan, M. Hossain, K.K. Su-Myat, P.L. Wood, K. Krenitsky, I. Takemasa, M. Miyake, M. Sekimoto, M. Monden, H. Matsubara, F. Nomura, D.B. Goodenowe, BMC Med. 8 (2010) 13.

[94] T. Hibi, T. Mori, M. Fukuma, K. Yamazaki, A. Hashiguchi, T. Yamada, M. Tanabe, K. Aiura, T. Kawakami, A. Ogiwara, T. Kosuge, M. Kitajima, Y. Kitagawa, M. Sakamoto, Clin. Cancer Res. 15 (2009) 2864.

[95] B.Sitek, B. Sipos, I. Alkatout, G. Poschmann, C. Stephan, T. Schulenborg, K. Marcus, J. Lüttges, D.D. Dittert, G. Baretton, W. Schmiegel, S.A. Hahn, G. Klöppel, H.E. Meyer, K. Stühler, J. Proteome Res. 8 (2009) 1647.

[96] K.H. Yu, C.G. Barry, D. Austin, C.M. Busch, V. Sangar, A.K. Rustgi, I.A. Blair, J. Proteome Res. 8 (2009) 1565.

[97] P.V. de Almeida, A.M. Grégio, M.A. Machado, A.A. de Lima, L.R. Azevedo, J. Contemp. Dent. Pract. 9 (2008) 72. 
[98] S. Nishiumi, M. Shinohara, A. Ikeda, T. Yoshie, N. Hatano, S. Kakuyama, S. Mizuno, T. Sanuki, H. Kutsumi, E. Fukusaki, T. Azuma, T. Takenawa, M. Yoshida, Metabolomics 6 (2010) 518.

[99] F. Fang, X. He, H. Deng, Q. Chen, J. Lu, M. Spraul, Y. Yu, Cancer. Sci. 98 (2007) 1678 .

[100] O.F. Bathe, R. Shaykhutdinov, K. Kopciuk, A.M. Weljie, A. McKay, F.R. Sutherland, E. Dixon, N. Dunse, D. Sotiropoulos, H.J. Vogel, Cancer Epidemiol. Biomarkers Prev. 20 (2011) 140.

[101] V. Iob, M. McMath, W.W. Coon, J. Surg. Res. 3 (1963) 85.

[102] L. Lavie, P. Lavie, J. Circadian Rhythms 2 (2004) 5.

[103] T. Uchikata, A. Matsubara, E. Fukusaki, T. Bamba, J. Chromatogr. A. 1250 (2012) 69.

[104] C. Deng, X. Zhang, N. Li, J. Chromatogr. B 808 (2004) 269.

[105] C.L. Silva, M. Passos, J.S. Câmara, Br. J. Cancer 105 (2011) 1894.

[106] A.W. Boots, J.J.B.N. van Berkel, J.W. Dallinga, A. Smolinska, E.F. Wouters, F.J. van Schooten, J. Breath Res. 6 (2012) 027108.

[107] S. Kakuta, Y. Bando, S. Nishiumi,M. Yoshida, E. Fukusaki, T. Bamba, Mass Spectrometry 2 (2013) A0018.

[108] S. Bijlsma, I. Bobeldijk, E.R. Verheij, R. Ramaker, S. Kochhar, I.A. Macdonald, B. van Ommen, A.K. Smilde, Anal. Chem. 78 (2006) 567.

[109] W.B. Dunn, D. Broadhurst, P. Begley, E. Zelena, S. Francis-McIntyre, N. Anderson, M. Brown, J.D. Knowles, A. Halsall, J.N. Haselden, A.W. Nicholls, I.D. Wilson, D.B. Kell, R. Goodacre, Nat. Protoc. 6 (2011) 1060.

[110] J. Xia, R. Mandal, I.V. Sinelnikov, D. Broadhurst, D.S. Wishart, Nucleic Acids Res. 40 (2012) W127.

[111] J. Zhang, J. Bowers, L. Liu, S. Wei, G.A.N. Gowda, Z. Hammoud, D. Raftery, PLoS One 7 (2012) e30181.

[112] H. Wu, R. Xue, C. Lu, C. Deng, T. Liu, H. Zeng, Q. Wang, X. Shen, J. Chromatogr. B 877 (2009) 3111.

[113] D. Djukovic, H.R. Baniasadi, R. Kc, Z. Hammoud, D. Raftery, Rapid Commun. Mass Spectrom. 24 (2010) 3057.

[114] H. Song, J.S. Peng, D.S. Yao, Z.L. Yang, H.L. Liu, Y.K. Zeng, X.P. Shi, B.Y. Lu, Brazilian J. Med. Biol. Res. 45 (2012) 78. 
[115] Z. Cai, J.S. Zhao, J.J. Li, D.N. Peng, X.Y. Wang, T.L. Chen, Y.P. Qiu, P.P. Chen, W.J. Li, L.Y. Xu, E.M. Li, J.P.M. Tam, R.Z. Qi, W. Jia, D. Xie, Mol. Cell. Proteomics 9 (2010) 2617.

[116] E.C.Y. Chan, P.K. Koh, M. Mal, P.Y. Cheah, K.W. Eu, A. Backshall, R. Cavill, J.K. Nicholson, H.C. Keun, J. Proteome Res. 8 (2009) 352.

[117] J.L. Chen, J. Fan, L.S. Yan, H.Q. Guo, J.J. Xiong, Y. Ren, J.D. Hu, Gastroenterol. Res. Pract. 2012 (2012) 125890.

[118] M. Mal, P.K. Koh, P.Y. Cheah, E.C.Y. Chan, Anal. Bioanal. Chem. 403 (2012) 483.

[119] F. Li, X. Qin, H. Chen, L. Qiu, Y. Guo, H. Liu, G. Chen, G. Song, X. Wang, F. Li, S. Guo, B. Wang, Z. Li, Rapid Commun. Mass Spectrom. 27 (2013) 24.

[120] L. Zhou, Q. Wang, P. Yin, W. Xing, Z. Wu, S. Chen, X. Lu, Y. Zhang, X. Lin, G. Xu, Anal. Bioanal. Chem. 403 (2012) 203.

[121] A.D. Patterson, O. Maurhofer, D. Beyoglu, C. Lanz, K.W. Krausz, T. Pabst, F.J. Gonzalez, J.F. Dufour, J.R. Idle, Cancer Res. 71 (2011) 6590.

[122] H. Wu, R. Xue, L. Dong, T. Liu, C. Deng, H. Zeng, X. Shen, Anal. Chim. Acta 648 (2009) 98 .

[123] S.Y. Liu, R.L. Zhang, H. Kang, Z.J. Fan, Z. Du, World J. Gastroenterol. 19 (2013) 3423.

[124] H. Cao, H. Huang, W. Xu, D. Chen, J. Yu, J. Li, L. Li, Anal. Chim. Acta 691 (2011) 68.

[125] S. Urayama, W. Zou, K. Brooks, V. Tolstikov, Rapid Commun. Mass Spectrom. 24 (2010) 613.

[126] P. Kaur, K. Sheikh, A. Kirilyuk, K. Kirilyuk, R. Singh, H.W. Ressom, A.K. Cheema, Int. J. Mass Spectrom. 310 (2012) 44.

\section{Figure captions}

Fig. 1. The omics cascade: the pathway from the gene to metabolism

Fig. 2. Outline of the experimental process required to produce metabolomic clinical screening tools 


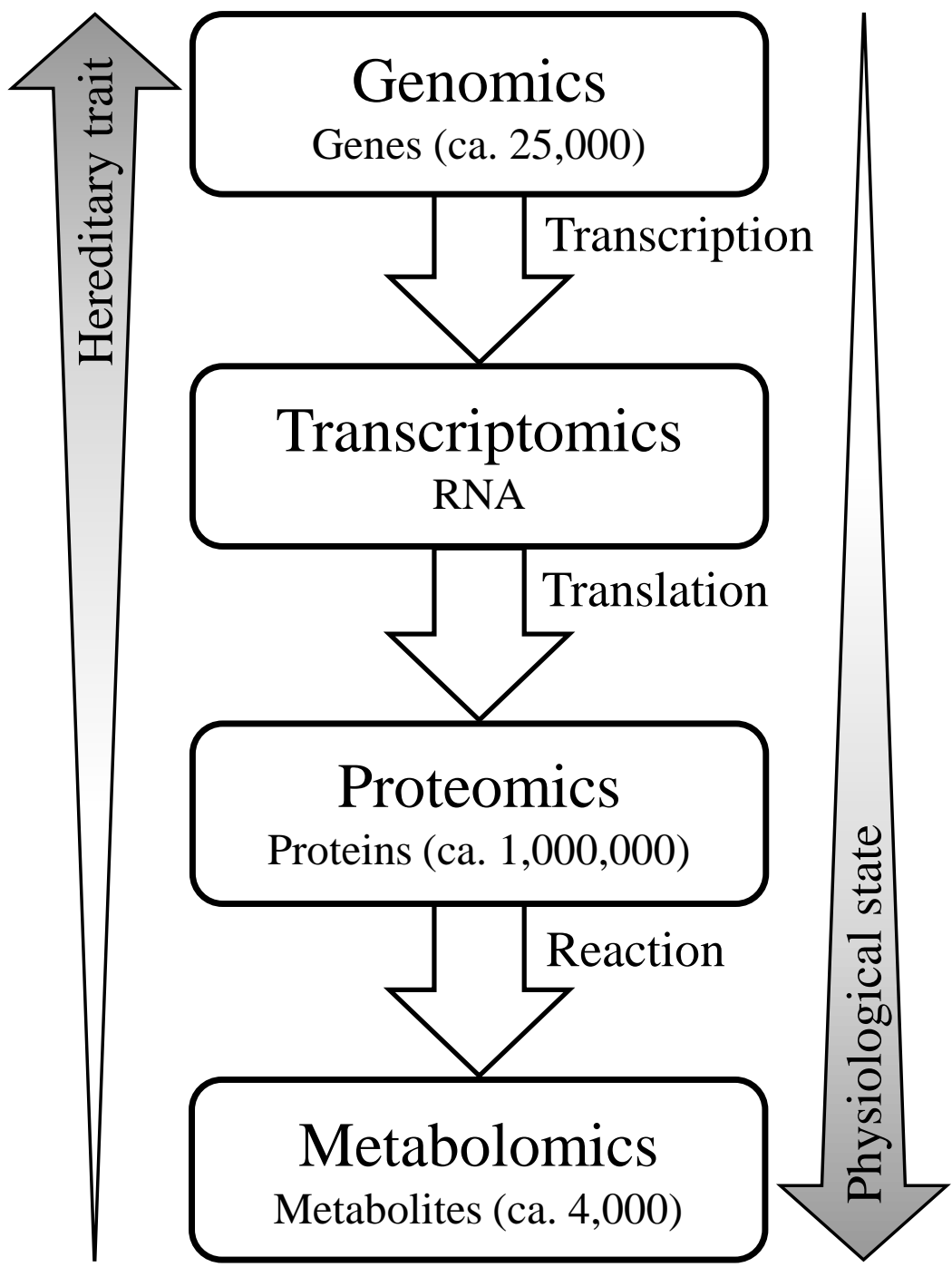




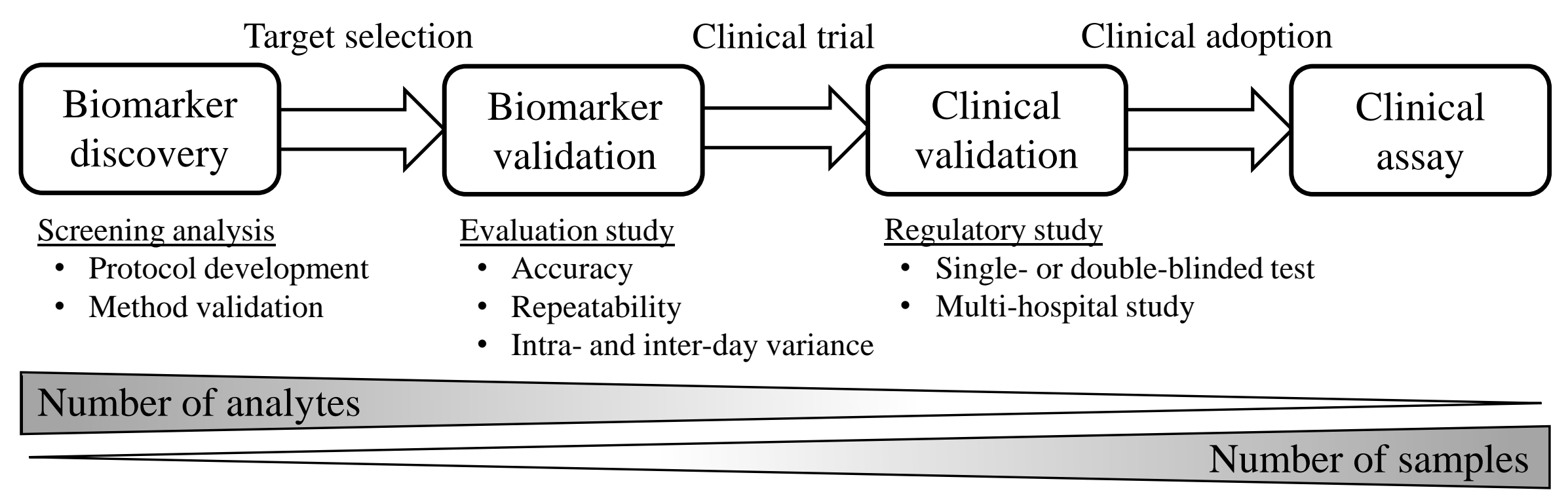


Table 1 Gastroenterological disease biomarker studies that employed metabolome analysis

\begin{tabular}{|c|c|c|c|c|c|}
\hline Disease & Specimen & Instrument & Upregulated metabolites & Downregulated metabolites & Reference \\
\hline Oral cancer & Saliva & UPLC-Q-TOF/MS & Lactate; $n$-Eicosanoate & $\begin{array}{l}\text { Valine; } \gamma \text {-Aminobutyrate; } \\
\text { Phenylalanine }\end{array}$ & [77] \\
\hline Oral cancer & Urine & GC-QMS & $\begin{array}{l}\text { Alanine; Valine; Serine; Tyrosine; } \\
\text { Cystine }\end{array}$ & 6-Hydroxynicotinate; Hippurate & [78] \\
\hline Oral cancer & Serum & GC-QMS & $\begin{array}{l}\text { Glycerate; Serine; Laurate; } \\
\text { N-Acetyl-L-aspartate; Asparagine; } \\
\text { Ornithine; Heptadecanoate }\end{array}$ & None & [80] \\
\hline Esophageal cancer & Serum & $\begin{array}{l}\text { LC-Q-TOF/MS } \\
\text { NMR }\end{array}$ & $\begin{array}{l}\text { Lactate; } \beta \text {-Hydroxybutyrate; Lysine; } \\
\text { Glutamine; Citrate }\end{array}$ & $\begin{array}{l}\text { Valine; Leucine/Isoleucine; } \\
\text { Methionine; Tyrosine; Tryptophan; } \\
\text { Myristate; Linoleate }\end{array}$ & [111] \\
\hline Esophageal cancer & $\begin{array}{l}\text { Mucosal } \\
\text { tissue }\end{array}$ & GC-QMS & $\begin{array}{l}\text { L-Valine; Naphthalene; 1-Butanamine; } \\
\text { Pyrimidine; Aminoquinoline; } \\
\text { L-Tyrosine; Isoleucine; Purine; Serine; } \\
\text { Phosphate; myo-Inositol; } \\
\text { Arabinofuranoside; L-Asparagine; } \\
\text { Tetradecanoate; L-Alanine; } \\
\text { Hexadecanoate }\end{array}$ & $\begin{array}{l}\text { L-Altrose; D-Galactofuranoside; } \\
\text { Arabinose; Bisethane }\end{array}$ & [112] \\
\hline Esophageal cancer & Urine & NMR & $\begin{array}{l}\text { Urea; Acetate; Pantothenate; } \\
\text { 3-Hydroxyisovaleate; Acetone; } \\
\text { Formate; 2-Hydroxyisobutyrate; } \\
\text { Creatinine; Ethanolamine; } \\
\text { 2-Aminobutyrate; Leucine; Succinate; } \\
\text { Glutamine; Glucose; Glycine; } \\
\text { Tryptophan; Trimethylamine- } N \text {-oxide; } \\
\text { Valine; Lactate; Tyrosine }\end{array}$ & Dimethylamine; Alanine; Citrate & [89] \\
\hline Esophageal cancer & Serum & LC-QqQ/MS & Uridine & $\begin{array}{l}\text { 1-Methyladenosine; } \\
N^{2}, N^{2} \text {-Dimethylguaosine; } \\
N^{2} \text {-Methylguanosine; Cytidine }\end{array}$ & [113] \\
\hline
\end{tabular}


Esophageal cancer

Serum

GC-QMS

Gastric cancer

Colorectal cancer

Gastric cancer

Serum

GC-QMS

Gastric cancer
Esophageal cancer: Lactate; Glycolate; Malonate; Fumarate; L-Serine;

L-Aspartate; L-Glutamine

Gastric cancer: 3-Hydroxypropionate; 3-Hydroxyisobutyrate

Colorectal cancer: Lactate; Glycolate; L-Alanine; 3-Hydroxypropionate;

L-Proline; L-Methionine;

Thioglycolate; L-Glutamate;

L-Asparagine; L-Glutamine;

Glucuronic lactone

L-Valine; Sarcosine; Hexadecanenitrile
Esophageal cancer: Pyruvate

Gastric cancer: Pyruvate; Octanoate;

Phosphate

Colorectal cancer: None

L-Glutamine; Hexanedioate;

9,12-Octadecadienoate;

9-Octadecenoate;

trans-13-Octadecenoate;

Nonahexacontanoate;

Cholesta-3,5-diene;

Cholesterol/Pentafluoropropionate;

Cholesterol; Cholest-5-en-3-ol;

Fumarate; 2-O-Mesyl arabinose;

Benzeneacetonitrile;

2-Amino-4-hydroxy-pteridinone;

1,2,4-Benzenetricarboxylate

Hydroxyacetate;

3,4-Dihydroxy-2(3H)-furanone;

3-Aminoisobutanoate; Valine;

2-Hydroxy-4-methyl-pentanoate;

Isoleucine; Proline; Uracil; Threonine;

Thymine; Dihydrouracil; Aspartate;

Pyroglutamate; GABA; Cysteine;

Nicotinamide; Glycerol phosphate;

Tetradecanoate; Palmitelaidate;

Palmitate; Linoleate; Stearate;

Glutamate; Dodecanoate; Asparagine; Sucrose; Cholesterol

Gluconate; Xanthine; $N$-Acetyl

glucosamine; Kynurenine; Inosine 


$\begin{array}{lll}\text { Colorectal cancer } & \begin{array}{l}\text { Mucosal } \\ \text { tissue }\end{array} & \text { NMR } \\ \text { GC-QMS }\end{array}$

Colorectal cancer

Colorectal cancer

Urine

Urine

Colorectal cancer

Tissue

GC×GC-TOF/MS

Colorectal cancer

Serum

FTICR-MS

LC-Q-TOF/MS

NMR

QqQ-MS

Colorectal cancer

DI-FTICR-MS
Lactate; Phosphate; L-Glycine;

2-Hydroxy-3-methylvalerate;

L-Proline; L-Phenylalanine; Palmitate;

Margarate; Oleate; Stearate; Uridine;

11,14-Eicosadienoate; 11-Eicosenoate;

1-O-Heptadecylglycerol;

1-Monooleoylglycerol; Propyl

octadecanoate; Cholesterol

Lactate; Arginine; Leucine; Isoleucine; Valine

5-Hydroxytryptophan;

5-Hydroxyindoleacetate; Tryptophan;

Glutamate; 5-Oxoproline;

$N$-Acetyl-aspartate; $p$-Cresol;

2-Hydroxyhippurate; Phenylacetate;

Phenylacetylglutamine;

p-Hydroxyphenylacetate

Glycine; L-Proline; L-Phenylalanine;

L-Alanine; L-Leucine; L-Valine;

L-Serine; L-Threonine; L-Isoleucine;

Picolinate; L-Methionine; L-Aspartate;

$\beta$-Alanine; Aminomalonate;

1-Methyl-hydantoin; Palmitate;

Margarate; Oleate; Stearate;

11-Eicosenoate; Myristate;

Pentadecanoate; Linolenate;

Lignocerate; Phosphate; L-Arabinose;

Lactate; Maleate; Pantothenate;

Glycerol; 1-Monooleoylglycerol;

Uracil; Uridine; Cholesterol

None

FA (C28H46O4, C28H48O4,

C28H50O4)

D-Mannose;

D-Galactose; D-Glucose; Fumarate;

Malate; Oxalate; Succinate; Ribitol;

Squalene

Succinate; Isocitrate; Citrate;

3-Methylhistidine; Histidine 


\section{Colorectal cancer $\quad$ Serum $\quad$ GC-QMS}

Hepatic cancer

Hepatic cancer

Hepatic cancer

Hepatic cancer

GC-QMS

Urine GC-QMS

\section{Tissue}

LPC(18:0); LPC(20:4); LPC(22:6); PC(34:1); LPA(16:0); LPA(18:0);

\section{LPC $(16: 0)$}

Pyruvate; 2-Hydroxybutyrate;

Phosphate; Isoleucine; $\beta$-Alanine; meso-Erythritol; Aspartate;

Pyroglutamate; Glutamate;

p-Hydroxybenzoate; Arabinose;

Asparagine; Xylitol; Ornithine;

Citrulline; Glucuronate; Glucosamine;

Palmitoleate; Inositol; Kynurenine;

Cystamine; Cystine; Lactitol

Serum LC-Q-TOF/MS

Cortisol; GCA; GCDCA; C16:1-CN;

FA (C16:1, C16:0, C18:2, C18:1,

C18:0, C20:5, C20:4, C20:2, C22:6,

\section{$\mathrm{C} 22: 5)$}

Plasma UPLC-QqQ/MS

LPC(24:0); Glycodeoxycholate;

Deoxycholate 3-sulfate

Octanedioate; Glycine; Tyrosine;

Threonine; Butanedioate
Arachidyl carnitine; Tetradecanal; Oleamide
Hexadecanedioate; Octadecanoate;

Eicosatrienoate; Myristate

Nonanoate; Creatinine; Ribulose;

$O$-Phosphoethanolamine

Tryptophan; LPC(0:0/14:0);

LPC(14:0/0:0); LPC(20:3);

LPC(20:5); C10-CN; C10:1-CN;

C8-CN; C6-CN

LPC(14:0); LPC(16:0); LPC(18:0);

LPC(18:1); LPC(18:2); LPC(18:3);

LPC(20:4); FA(24:0); FA(24:1);

$\operatorname{LPC}(20: 2) ; \operatorname{LPC}(20: 3) ; \operatorname{LPC}(20: 5)$

Heptanedioate; Ethanedioate; Xylitol;

Urea; Phosphate; Propanoate;

Pyrimidine; Butanoate;

Trihydroxypentanoate;

Hypoxanthine; Arabinofuranose;

Hydroxyproline dipeptide; Xylonate

Sitosterol- $\beta$; L-Phenylalanine;

LPC (18:2(9Z, 12Z));

Glycerophosphocholine;

LPE(18:3(9Z, 12Z, 15Z)/0:0);

Chenodeoxycholate glycine

conjugate; LPC(22:6(4Z, 7Z, 10Z,

13Z, 16Z, 19Z)); Quinaldate;

LPE(18:0/0:0); LPC(18:0);

LPC(20:4(5Z, 8Z, 11Z, 14Z)) 


\begin{tabular}{|c|c|c|c|}
\hline Hepatic cancer & Feces & UPLC-Q-TOF/MS & LPC(18:0); LPC(16:0) \\
\hline Pancreatic cancer & Plasma & $\begin{array}{l}\text { GC-TOF/MS } \\
\text { LC-IT/MS } \\
\text { LC-LTQ-Orbitrap-MS }\end{array}$ & $\begin{array}{l}\text { Arachidonate; Erythritol; Cholestero } \\
N \text {-Methylalanine; Lysine; } \\
\text { Deoxycholylglycine; Cholylglycine; } \\
\text { LPC(16:0); Tauroursodeoxycholate; } \\
\text { Taurocholate; LPC(18:2); PE(26:0); } \\
\text { PC (34:2) }\end{array}$ \\
\hline Pancreatic cancer & Serum & GC-QMS & $\begin{array}{l}\text { Lactate; Thiodiglycolate; } \\
\text { 7-Hydroxyoctanoate; Asparagine; } \\
\text { Aconitate; Homogentisate; } \\
N \text {-Acetyltyrosine }\end{array}$ \\
\hline Pancreatic cancer & Tissue & UPLC-TOF/MS & Taurine \\
\hline
\end{tabular}

Chenodeoxycholate dimeride;

Urobilin; Urobilinogen

7-Ketolithocholate

Glutamine; Hydrocinnamate;

Phenylalanine; Tryptamine; Inosine

Glycine; Urea, Octanoate; Glycerate;

Decanoate; Laurate; Myristate;

Palmitate; Urate; Margarate; Stearate

Succinate; Malate; Uridine;

Glutathione;

UDP- $N$-acetyl-D-glucosamine; NAD;

UMP; AMP

GABA, $\gamma$-Aminobutyric acid; LPC, Lysophosphatidylcholine; PC, Phosphatidylcholine; LPA, Lysophosphatidic acid; LPE, Lysophosphatidylethanolamine; PE, Phosphatidylethanolamine; FA, Fatty acids; GCDCA, Glycochenodeoxycholic acid; GCA, Glycocholic acid; UDP, Uridine diphosphate; NAD,

Nicotinamide adenine dinucleotide; UMP, Uridine monophosphate; AMP, Adenosine monophosphate 
Table 2 Data processing software for metabolome analysis

\begin{tabular}{|c|c|c|c|c|}
\hline Software & Experimental data & User interface & Application & Reference \\
\hline MetAlign & $\begin{array}{l}\text { LC-MS } \\
\text { GC-MS }\end{array}$ & $\begin{array}{l}\text { Local application } \\
\text { (Graphical user interface) }\end{array}$ & $\begin{array}{l}\text { Format conversion } \\
\text { Baseline correction } \\
\text { Peak detection } \\
\text { Peak alignment } \\
\text { Peak filtering }\end{array}$ & [60] \\
\hline XCMS & $\begin{array}{l}\text { LC-MS } \\
\text { GC-MS } \\
\text { LC-MS/MS }\end{array}$ & Web-based platform & $\begin{array}{l}\text { Peak detection } \\
\text { Peak alignment } \\
\text { Peak filtering } \\
\text { Data visualization }\end{array}$ & [61] \\
\hline MZmine & LC-MS & $\begin{array}{l}\text { Local application } \\
\text { (Graphical user interface) }\end{array}$ & $\begin{array}{l}\text { Peak detection } \\
\text { Peak alignment } \\
\text { Peak filtering } \\
\text { Deisotoping } \\
\text { Data visualization }\end{array}$ & [62] \\
\hline AIoutput & GC-MS & $\begin{array}{l}\text { Local application } \\
\text { (Microsoft Excel VBA) }\end{array}$ & $\begin{array}{l}\text { Peak identification } \\
\text { Data visualization }\end{array}$ & [63] \\
\hline MRMPROBS & LC-MS/MS & $\begin{array}{l}\text { Local application } \\
\text { (Graphical user interface) }\end{array}$ & $\begin{array}{l}\text { Peak detection } \\
\text { Peak filtering } \\
\text { Peak identification } \\
\text { Data visualization }\end{array}$ & [64] \\
\hline
\end{tabular}

